Artículo

\title{
Propiedades tecnológicas y fisicoquímicas de la leche y características fisicoquímicas del queso Oaxaca tradicional
}

Eric Montes de Oca-Flores ${ }^{\mathrm{a}}$

Angélica Espinoza-Ortega ${ }^{\mathrm{a}}$

Carlos Manuel Arriaga-Jordán ${ }^{\mathrm{a}}$

${ }^{a}$ Instituto de Ciencias Agropecuarias y Rurales (ICAR, Universidad Autónoma del Estado de México). Carretera a Tlachaloya SN, Cerrillo Piedras Blancas 50090 Toluca de Lerdo, México.

*Autor de correspondencia: angelica.cihuatl@gmail.com

\section{Resumen:}

Los parámetros tecnológicos de la leche (características tecnológicas y fisicoquímicas) son de gran importancia porque influyen en el rendimiento y calidad del queso. Se sabe que los parámetros en leche varían dependiendo de la época del año, pero no se ha estudiado su efecto en queso en sistemas tradicionales. El objetivo del trabajo fue evaluar las características tecnológicas y fisicoquímicas de la leche, así como las fisicoquímicas del queso Oaxaca tradicional en época de secas y lluvias. Muestras de leche y queso se tomaron de 21 diferentes queserías a pequeña escala. En leche se analizó porcentaje de grasa $(\mathrm{G})$, proteína (P) y acidez, tiempo de coagulación (TC), firmeza de la cuajada (FC) y rendimiento (RTO). En queso se analizó G, P, acidez, humedad y cloruros. Se utilizó un ANOVA de un factor $(P<0.05)$, para evaluar las variaciones por estación. Se observaron diferencias significativas por época en G, P y acidez, además del TC, FC y RTO en leche; y G, acidez y humedad en queso. Los resultados del estudio muestran que las diferencias en las características fisicoquímicas y tecnológicas de la leche por época del año, se ven reflejadas en la composición del queso.

Palabras clave: Queso Oaxaca, Leche, Tradicional, Propiedades tecnológicas, Propiedades fisicoquímicas. 
Recibido: 04/10/2016

Aceptado: 08/05/2018

\section{Introducción}

La composición de la leche cruda está determinada por diversos factores, tales como la raza, alimentación, época del año, etapa de lactancia ${ }^{(1,2,3)}$; diferencias que son de gran importancia en la elaboración de los productos lácteos (quesos), en particular por su efecto en las propiedades tecnológicas de la leche reflejadas en el rendimiento y calidad del queso ${ }^{(4,5)}$, por el tiempo de coagulación (TC) y la firmeza del gel (F).

Una mayor concentración de grasa y proteína aunada a parámetros tecnológicos favorables, permiten menor tiempo de coagulación y una mayor firmeza de la cuajada ${ }^{(4)}$. Por lo tanto, existe una correlación positiva entre los porcentajes de grasa y proteína de la leche con el rendimiento de queso; es decir a mayor concentración de sólidos, mayores rendimientos ${ }^{(6)}$. Por otro lado, la calidad y rendimiento quesero no es solo por volumen, sino por la cantidad y calidad de la proteína ${ }^{(7)}$. La proteína es un componente importante en los quesos, por su influencia en los parámetros tecnológicos ${ }^{(8)}$, principalmente la $\mathrm{K}$ caseína $^{(9,10)}$, tal como lo han reportado algunos trabajos sobre la buena coagulación de la leche con altos porcentajes de k-caseína, además del contenido de calcio y el $\mathrm{pH}^{(11,12)}$.

En relación con el clima, algunos autores mencionan que son uno de los factores importantes y ejercen gran influencia en la calidad del queso ${ }^{(13,14)}$, debido a las variaciones en el porcentaje de grasa, proteína y lactosa de la leche a lo largo del año, siendo menores en primavera-verano y mayores en otoño-invierno; variación explicada principalmente por cambios en la dieta de las vacas ${ }^{(15)}$. No obstante esos trabajos se han realizado en otras regiones del mundo, pero en los quesos mexicanos es necesario su estudio.

En la zona centro de México se elabora de manera artesanal el queso Oaxaca, un producto tradicional perteneciente al grupo de pasta fillata, cuyo nombre es atribuido a su lugar de origen, y es el más conocido en el país. Es un queso fresco, en su elaboración la cuajada se somete a un amasado en agua caliente hasta estirarse y formar bandas que posteriormente se enredan en forma de madejas ${ }^{(16)}$. Se obtiene a partir de leche cruda de vaca, que como ya se mencionó, tiene variabilidad por aspectos atribuidos al animal y externos $^{(17,18,19)}$ y cuya composición y propiedades tecnológicas influyen en el valor nutricional de los productos lácteos ${ }^{(15)}$, particularmente los quesos elaborados a partir de leche cruda ${ }^{(20)}$.

Hasta la fecha se han realizado estudios sobre el queso Oaxaca que documentan el proceso de elaboración y las características fisicoquímicas ${ }^{(21)}$, y la relación entre el sabor y la textura del queso ${ }^{(22)}$; sin embargo es pertinente analizar el efecto de la calidad de la leche sobre el queso en diferentes épocas del año, en virtud que estudios realizados en sistemas 
de producción de leche en pequeña escala que abastecen a las queserías productoras de queso Oaxaca, encontraron diferencias significativas $(P<0.05)$ en porcentaje de grasa y densidad en diferentes periodos del año ${ }^{(23)}$. Dado que este queso se elabora todo el año, existe la necesidad de documentar si esa variación en leche ejerce algún efecto en el rendimiento y calidad del queso. Por lo tanto, esta investigación tuvo como propósito evaluar las propiedades tecnológicas y fisicoquímicas de la leche, y su correlación, así como las características físicoquímicas del queso Oaxaca tradicional en época de secas y lluvias.

\section{Material y métodos}

\section{Muestras de queso y leche}

El trabajo se llevó a cabo en queserías de pequeña escala en la zona nororeste del Estado de México. Se obtuvieron directamente de las queserías ${ }^{(23)}$ muestras de leche y queso (por triplicado). La época de secas comprendió de febrero-abril, y de agosto-octubre la de lluvias. Las muestras se transportaron a $4{ }^{\circ} \mathrm{C}$ y los análisis se realizaron antes de las $24 \mathrm{~h}$ del muestreo.

\section{Análisis en leche}

Fisicoquímicos: se analizó grasa $(\mathrm{G})$, proteína $(\mathrm{P})$, mediante ondas de ultrasonido con un analizador Eco-milk Analyzer KAM98-2 ${ }^{\mathrm{a}(21)}$ y acidez por medio de NAOH 0.1 M/fenolftaleína como indicador.

Tecnológicos: Se determinaron tiempo de coagulación (TC), firmeza de la cuajada (FC) y rendimiento (RTO); para cada prueba las muestras se calentaron a $35{ }^{\circ} \mathrm{C}$ e inmediatamente se les adicionó el $10 \%$ de cuajo (cuajo industrial), manteniendo temperatura en baño maría. El TC se realizó detectando el inicio de la coagulación al producirse un aumento brusco de la viscosidad ${ }^{(24)}$, para lo cual se utilizó un viscosímetro Brookfield (Engineering labs. INC. Middleboro, MA 02346. USA) modelo LVT, con espina del No. 1 a una velocidad de $12 \mathrm{rpm}$. La FC se midió 30 min después de la adición del cuajo, calculándose la fuerza $(\mathrm{N})$ de compresión ${ }^{(25)}$ con un Texturometro TX-XT2, sonda cilíndrica de $10 \mathrm{~mm}$ de diámetro, ciclo de compresión de $20 \mathrm{~mm}$ de profundidad y velocidad de $1 \mathrm{~mm} / \mathrm{seg}$. El RTO se determinó por centrifugación para forzar la liberación 
de suero; en tubos eppendorf se adicionó $1 \mathrm{ml}$ de muestra (leche a $35{ }^{\circ} \mathrm{C}$ con el $10 \%$ de cuajo), después de $30 \mathrm{~min}$ se centrifugó a $14,000 \mathrm{rpm}$ por $30 \mathrm{~min}$ a $35^{\circ} \mathrm{C}^{(26)}$; el rendimiento de queso se calculó por la diferencia del peso inicial de la muestra con el de la cuajada. Para este proceso se utilizó una centrifuga (Universal 320R. D-7852Tuttlingen Heftich).

\section{Análisis en queso}

La acidez se determinó por medio de NAOH 0.1 M/fenolftaleína como indicador ${ }^{(21)}$; los métodos oficiales de la AOAC (1990) para grasa (933.05), proteína (991.20) y humedad (926.08); $\mathrm{NaCl}$ por el método Volhard ${ }^{(27)}$.

\section{Análisis estadístico}

Para analizar los resultados de leche y queso, se realizó un análisis de varianza de un factor (época) y las diferencias existentes se calcularon mediante la prueba de Tukey $(P<0.05)$. La relación entre las propiedades fisicoquímicas y tecnológicas de la leche se analizaron mediante una correlación de Pearson, con el paquete estadístico STATGRAPHICS Plus.

\section{Resultados y discusión}

\section{Propiedades fisicoquímicas y tecnológicas de la leche}

Las propiedades fisicoquímicas (Cuadro 1) por época presentaron diferencias significativas $(P<0.05)$ para el contenido de grasa, proteína y acidez, con valores mayores de grasa y menores en proteína en época de lluvias. Estudios previos ${ }^{(23)}$ que evaluaron la calidad fisicoquímica de leches, encontraron diferencias sólo en el porcentaje de grasa y densidad, con valores mayores para grasa en los periodos que comprenden los meses de lluvias (promedio $3.62 \%$ ), resultados similares a los obtenidos en este trabajo. Trabajos realizados en los meses de junio a octubre ${ }^{(21)}$ reportan porcentajes de 3.34 y 3.05 de grasa y proteína respectivamente; otro trabajo obtuvo en promedio 3.18 en grasa y 2.97 en proteína $^{(28)}$, resultados muy por debajo de los obtenidos en el presente estudio. 
Los cambios en las propiedades fisicoquímicas y tecnológicas de la leche en las diversas estaciones, son consecuencia de la disponibilidad de forrajes en la alimentación del ganado. Algunos autores reportan valores mayores de grasa y proteína en época de lluvias, cuando la alimentación de los animales estuvo conformada principalmente por forrajes verdes, entre ellos trébol ${ }^{(29,30)}$.

Cuadro 1: Análisis de medias de las propiedades fisicoquímicas y tecnológicas de la leche en época de lluvias y secas

\begin{tabular}{lcccccc}
\hline I & $\begin{array}{c}\text { Grasa } \\
(\boldsymbol{\%})\end{array}$ & $\begin{array}{c}\text { Proteína } \\
(\boldsymbol{\%})\end{array}$ & $\begin{array}{c}\text { Acidez } \\
\left({ }^{\mathbf{0}} \mathbf{D}\right)\end{array}$ & $\begin{array}{c}\text { TC } \\
(\text { minutos })\end{array}$ & $\begin{array}{c}\text { FC } \\
(\text { Newton) }\end{array}$ & $\begin{array}{c}\text { RTO } \\
(\%)\end{array}$ \\
\hline Secas & $3.28^{\mathrm{a}}$ & $3.02^{\mathrm{a}}$ & $23.6^{\mathrm{a}}$ & $16.7^{\mathrm{a}}$ & $0.11^{\mathrm{a}}$ & $12.6^{\mathrm{a}}$ \\
Lluvias & $3.64^{\mathrm{b}}$ & $2.94^{\mathrm{b}}$ & $23.1^{\mathrm{b}}$ & $27.0^{\mathrm{b}}$ & $0.09^{\mathrm{b}}$ & $10.8^{\mathrm{b}}$ \\
& 0.014 & 0.004 & 0.024 & 0.565 & 0.001 & 0.082 \\
\hline
\end{tabular}

$\mathrm{TC}=$ tiempo de coagulación $; \mathrm{FC}=$ firmeza de la cuajada $\mathrm{RTO}=$ rendimiento $;{ }^{\circ} \mathrm{D}=$ grados Dormic .

$\mathrm{EEM}=$ Error estándar de la media.

${ }^{\mathrm{ab}}$ Literales diferentes indican diferencias estadísticas $(P<0.001)$.

El TC, FC y RTO presentaron diferencias significativas $(P<0.001)$, con menor tiempo de coagulación, mayor firmeza y rendimiento en el periodo de secas. Si bien un porcentaje alto de grasa y proteína se relaciona con propiedades tecnológicas deseables (menores TC y mayor FC), además de altos $\mathrm{RTO}^{(23)}$, existen otros factores como el contenido y calidad de la proteína (contenido de k-caseína, las características de las micelas, polimorfismo genético $)^{(31-34)}$, además del contenido de calcio y $\mathrm{pH}^{(11,12)}$, que influyen en las propiedades tecnológicas, y que sería necesario analizar a detalle en un trabajo posterior.

La correlación RTO, FC, TC con la proteína fue mejor en la época de secas, y para grasa sólo fue positiva para RTO. Otros trabajos reportan rendimientos mayores con tiempos de coagulación menores a concentraciones altas de proteína y bajas en grasa ${ }^{(9)}$; en otro estudio obtuvieron menores tiempos de coagulación y mejores firmezas en invierno (14.61 min y $62.63 \mathrm{~mm}$, respectivamente), que en el verano (16.84 $\min$ y $59.33 \mathrm{~mm}$, respectivamente) ${ }^{(35)}$, situación dada posiblemente por presentar valores altos de grasa en el invierno.

En un trabajo llevado a cabo en un sistema tradicional ${ }^{(36)}$, se reportaron en invierno tiempos de coagulación de 6.06 min, con valores de proteína de 3.41 y de 4.17 en grasa; y para el verano de $3.26 \mathrm{~min}$, con valores de 3.48 y 3.93 para proteína y grasa respectivamente. Otros estudios ${ }^{(37,38)}$ establecen que si los tiempos de coagulación son mayores a $30 \mathrm{~min}$, la leche se considera como no apta para la producción de queso. En el presente trabajo el $14 \%$ de las muestras del periodo de secas y el $33 \%$ de las de lluvias presentaron tiempos de coagulación mayores a $30 \mathrm{~min}$. El forraje verde reduce el tiempo 
de coagulación enzimática de la leche, principalmente por su efecto en la proteína de la leche, tanto de caseínas como en proteínas del suero ${ }^{(36)}$; probablemente esa es la razón de los resultados en época de lluvias.

\section{Correlación entre las propiedades fisicoquímicas y tecnológicas de la leche}

La FC y el RTO en ambas épocas (Cuadros 2 y 3), presentaron una correlación negativa con el TC. Resultados similares se reportan en otro trabajo ${ }^{(26)}$, apuntando que el gel obtenido es débil cuando el tiempo de coagulación de la leche es mayor.

La acidez presentó una correlación negativa con el TC. Otros trabajos ${ }^{(23,39)}$ mencionan que a medida que aumenta la acidez, disminuyen los tiempos de coagulación y aumenta la firmeza de la cuajada; Así mismo otros reportes ${ }^{(39)}$ obtuvieron tiempos menores de coagulación a pH bajos; indicativo de la capacidad de coagulación que ejerce el pH bajo de la leche ${ }^{(37)}$.

Cuadro 2: Coeficiente de correlación entre las propiedades fisicoquímicas y tecnológicas de la leche en periodo de secas

\begin{tabular}{llll}
\hline & TC & FC & RTO \\
\hline Grasa & -0.149 & 0.169 & $0.388^{* * *}$ \\
Proteína & $-0.233^{* *}$ & $0.329 * * *$ & $0.286^{* *}$ \\
Acidez & $-0.534 * * *$ & $0.441^{* * *}$ & 0.032 \\
TC & 1.000 & $-0.608 * * *$ & $-0.250^{* *}$ \\
FC & $-0.608^{* * *}$ & 1.000 & 0.104 \\
RTO & $-0.250 * *$ & 0.104 & 1.000 \\
\hline
\end{tabular}

$\mathrm{TC}=$ tiempo de coagulación $; \mathrm{FC}=$ firmeza de la cuajada; $\mathrm{RTO}=$ rendimiento.

$* * P<0.01 ; * * * P<0.001$.

En relación con las correlaciones de los sólidos totales (grasa y proteína) con el TC, FC y RTO fueron muy variadas en ambas épocas (Cuadro 3). La grasa presentó correlación positiva solo con el RTO en ambas épocas y la proteína únicamente en secas. Sin embargo, se ha reportado que al aumentar el porcentaje de caseínas y de proteína, la firmeza de la cuajada es mejor, tal como se ha reportado en otros estudios ${ }^{(26,38,40)}$. 
Cuadro 3: Coeficiente de correlación entre las propiedades fisicoquímicas y tecnológicas de la leche en época de lluvias

\begin{tabular}{llll}
\hline & TC & \multicolumn{1}{c}{ FC } & \multicolumn{1}{c}{ RTO } \\
\hline Grasa & -0.030 & 0.067 & $0.328^{* * *}$ \\
Proteína & $-0.203^{*}$ & -0.069 & 0.08 \\
Acidez & $-0.660^{* * *}$ & 0.121 & 0.039 \\
TC & 1.000 & $-0.576^{* * *}$ & $-0.405 * * *$ \\
FC & $-0.576^{* * *}$ & 1.000 & $0.350^{* * *}$ \\
RTO & $-0.405^{* * *}$ & $0.350^{* * *}$ & 1.000 \\
\hline
\end{tabular}

$\mathrm{TC}=$ tiempo de coagulación $; \mathrm{FC}=$ firmeza de la cuajada; $\mathrm{RTO}=$ rendimiento.

$$
* P<0.05 ; * * * P<0.001 \text {. }
$$

Otro estudio ${ }^{(41)}$ documentó la correlación de varios componentes de la leche (G, P, ST, calcio, entre otros) con la firmeza de la cuajada y el tiempo de bifurcación de la cuajada, pero no para el tiempo de coagulación. A pesar de no coincidir las correlaciones entre épocas, se cumplen las disposiciones reportadas por diferentes autores. Estas diferencias se pueden atribuir a las condiciones de alimentación, como ya se mencionó.

\section{Propiedades fisicoquímicas del queso Oaxaca tradicional por época del año}

Se presentaron diferencias significativas $(P<0.05)$ en el porcentaje de grasa, siendo mayor en época de lluvias, al igual que la acidez y humedad (Cuadro 4). En una evaluación realizada a queso Ricotta y Pecorino, el porcentaje de grasa y proteína en leche no presentaron diferencias significativas en los periodos evaluados (primavera, verano, otoño e invierno). Sin embargo, el queso Ricotta si presentó diferencias $(P<0.05)$ en grasa, proteína y humedad ${ }^{(42)}$. En este estudio sólo el porcentaje de grasa en leche-queso se relacionó proporcionalmente, mayor en lluvias y menor en secas, no así para la proteína, pero con un mayor rendimiento en secas (12.6\%). Si bien la relación de sólidos en leche-queso, son significativos en las características finales del producto, también son importantes en el rendimiento; en ese sentido el rendimiento del queso está directamente relacionado con el nivel de grasa y caseína ${ }^{(43)}$. Para efectos de este trabajo los resultados muestran que la proteína es la que influye directamente en el rendimiento. 
Cuadro 4: Análisis de las características fisicoquímicas del queso por época

\begin{tabular}{lccccc}
\hline & $\begin{array}{c}\text { Grasa } \\
(\boldsymbol{\%})\end{array}$ & $\begin{array}{c}\text { Proteína } \\
(\mathbf{\%})\end{array}$ & $\begin{array}{c}\text { Acidez } \\
(\boldsymbol{\%})\end{array}$ & $\begin{array}{c}\text { Humedad } \\
(\boldsymbol{\%})\end{array}$ & $\begin{array}{c}\text { Cloruros } \\
(\boldsymbol{\%})\end{array}$ \\
\hline Secas & $20.6^{\mathrm{a}}$ & 18.5 & $0.8^{\mathrm{a}}$ & $50.4^{\mathrm{a}}$ & 4.2 \\
Lluvias & $22.2^{\mathrm{b}}$ & 18.7 & $1.0^{\mathrm{b}}$ & $51.3^{\mathrm{b}}$ & 4.3 \\
EEM & 0.103 & 0.078 & 0.011 & 0.104 & 0.067 \\
& & & & & \\
\hline
\end{tabular}

$\mathrm{EEM}=$ error estándar de la media.

ab Literales diferentes indican diferencias estadísticas $(P<0.05)$.

\section{Conclusiones e implicaciones}

Los resultados mostraron que la época del año, ejerció un efecto significativo en las características fisicoquímicas y o tecnológicas de la leche, y la composición del queso, principalmente por la disposición de alimento que se tiene en las diferentes épocas. Si bien los porcentajes de grasa, proteína y acidez, se correlacionan con las propiedades tecnológicas de la leche, la proteína tuvo un mayor efecto en el rendimiento quesero, reflejándose en quesos con bajo contenido de grasa y humedad. Las variaciones de la calidad de la leche y de los quesos al interior de las queserías, es una de las características de productos tradicionales que no cuentan con procesos estandarizados. Con estos trabajos se establecen las pautas tecnológicas que dan muestra de ello, considerándolas como parte de un proceso artesanal y que es parte de su tipicidad.

\section{Agradecimientos}

Esta investigación fue apoyada por el proyecto "Programa de desarrollo en la integración y agregación de valor en los eslabones de la cadena productiva caso: quesos mexicanos genuinos". Financiado por SAGARPA-CONACYT con clave: 1928/2011C. Un reconocimiento especial a los productores de queso de Aculco que colaboraron en este trabajo; que fue posible gracias a los fondos del Consejo Mexicano de Ciencia y Tecnología (CONACYT), y a la Universidad Autónoma del Estado de México (UAEM). Al CONACYT por la beca otorgada a Eric Montes de Oca Flores para llevar sus estudios de Doctorado. 


\section{Literatura citada:}

1. Dillon P, Buckley F, O'Connor P, Hegarty D, Rath M. A comparison of different dairy cow breeds on a seasonal grass-based system of milk production 1. Milk production, live weight, body condition score and DM intake. Livest Prod Sci 2003;(83):21-33.

2. Ozrenk E, Selcuk-Inci S. The effect of seasonal variation on the composition of cow milk in Van Province. Pak J Nut 2008;7(1):61-164.

3. Křřžová L, Hanuš O, Hadrová S, Kučera J, Samková E, Roubal P, Veselý A. Composition, physical and technological properties of raw milk as affected by cattle breed, season and type of diet. Ann Anim Sci 2014;(14):721-736.

4. Auldist, MJ, Johnston KA, White NJ, Fitzsimons WP, Boland MJA. Comparison of the composition, coagulation characteristics and cheesemaking capacity of milk from Friesian and Jersey dairy cows. J Dairy Res 2004;(71):51-57.

5. Schönfeldt HC, Hall NG, Smit LE. The need for country specific composition data on milk. Food Res Inter 2012;(47):207-209.

6. Barrón LJR, Fernández LE, Perea S, Chávarri F, María SVC, Torres MI, et al. Seasonal changes in the composition of bulk raw ewe's milk used for Idiazabal cheese manufacture. Int Dairy J 2001;(11):771-778.

7. Wedholm A, Larsen LB, Lindmark-Mansson H, Karlsson AH, Andren A. Effect of protein composition on the cheese-making properties of milk from individual dairy cows. J Dairy Sci 2006;(89):3296-3305.

8. Guinee TP. Role of protein in cheese and cheese products. In: Fox PF, McSweeney PLH editors. Advanced dairy chemistry 1: Proteins, Part B, 3rd ed. Kluver Academic/Plenum Publisher; 2003:1083-1174.

9. Alipanah M, Kalashnikova LA. Influence of k-casein genetic variant on cheese making ability. J Anim Vet Adv 2006;(7):855-857.

10. Jõudu I, Henno M, Värv S, Viinalass H, Püssa T, Kaart T, Arney D, Kärt O. The effect of milk proteins on milk coagulation properties in stonian dairy breeds. Vet ir Zootec 2009;(68):14-19.

11. Serrano MB, Garzón SAI, González AME, Oliver AF, Figueroa SA, Martínez HJ. Factores que influyen en el tiempo de coagulación y en la cuajada de leche de oveja de raza merina. Prod Ovi Capr 1998;(23):175-178.

12. Joudu I, Merike H, Tanel K, Tonu P, Olav K. The effect of milk protein contents on the rennet coagulation properties of milk from individual dairy cows. Int Dairy $\mathrm{J}$ 2008;(18):964-967. 
13. Mendina C, Ibánez FC, Torre P, Barcina Y. Influence of the season on proteolysis and sensory characteristics of idiazabal cheese. J Dairy Sci 2000;(83):1899-1904.

14. Lucey JA, Johnson ME, Horne DS. Perspective on the basis of the rheology and texture properties of cheese. J Dairy Sci 2003;(86):2725-2743.

15. Heck JML, Van-Valenberg HJF, Dijkstra J, Van-Hooijdonk ACM. Seasonal variation in the Dutch bovine raw milk composition. J Dairy Sci 2009;(92):47454755.

16. Cervantes EF, Villegas de Gante A. La leche y los quesos artesanales. $1^{\mathrm{a}}$ ed. México, DF: Editorial Porrúa; 2012.

17. Galina MA, Osnaya F, Cuchillo HM, Haenlein GFW. Cheese quality from milk of grazing or indoor fed Zebu cows and Alpine crossbred goats. Small Ruminant Res 2007;(71):264-272.

18. Martin B, Pomiés D, Pradel P, Verdier-Metz I, Rémond B. Yield and sensory properties of cheese made with milk from Holstein or Montbéliarde cows milked twice or once daily. J Dairy Sci 2009;(92):4730-4737.

19. Jaramillo DP, Buffa MN, Rodríguez M, Pérez-Baena I, Guamis B, Trujillo AJ. Effect of the inclusion of artichoke silage in the ration of lactating ewes on the properties of milk and cheese characteristics during ripening. J Dairy Sci 2010;(93):1412-1419.

20. Najera AI, Barrón LJR, Ribeiro P, Pelissier F, Abilleira E, Perez-Elortondo FJ, et al. Seasonal changes in the technological and compositional quality of ewe's raw milks from commercial flocks underpart-time grazing. J Dairy Res 2009;(76):301-307.

21. Montes de Oca-Flores E, Castelán-Ortega OA, Estrada-Flores JG, Espinoza-Ortega A. Oaxaca cheese: Manufacture process and physicochemical characteristics. Int J Dairy Technol 2009;(62):535-540.

22. Villanueva-Carvajal A, Esteban-Chávez M, Espinoza-Ortega A, Arriaga-Jordán CM, Dominguez-Lopez A. Oaxaca cheese: flavour, texture and their interaction in a Mexican traditional pasta filata type cheese. CyTA - J Food 2012;(1):63-70.

23. De Marchi M, Dal-Zotto R, Cassandro M, Bittante G. Milk coagulation ability of five dairy cattle Breeds. J Dairy Sci 2007;(90):3986-3992.

24. López MB, Laencina J. Viscosimetric measurement of milk clotting time. Sci Tec Lattiero - Casearia 1994;(45):169-182.

25. Berruga MI, Molina P, Román M, Molina A. Características del yogur de oveja según el tipo de fermento lácteo. Universidad de Castilla La Mancha. 2003. www.seoc.eu. Consultado 9 Mar, 2012.

26. Jaramillo P, Zamora A, Guamisa B, Rodríguez M, Trujillo AJ. Cheesemaking aptitude of two Spanish dairy ewe breeds: Changes during lactation and relationship 
between physico-chemical and technological properties. Small Ruminant Res 2008;(78):48-55.

27. NMX-F-360. Alimentos para humanos. Determinación de cloruros como cloruro de sodio (método de volhard). Foods for humans. Determination of chlorides as sodium chloride (volhard method), México. 1981.

28. Vázquez FC. Caracterización del queso Botanero producido en Aculco Estado de México [tesis maestría]. México: Universidad Autónoma del Estado de México; 2008.

29. Nobrega DB, Langoni H. Breed and season influence on milk quality parameters and in mastitis occurrence. Pesquisa Vet Brasil 2011;31(12):1045-1052.

30. Hattem HE, Taleb AT, Manal AN, Hanaa SS. Effect of pasteurization and season on milk composition and ripening of Ras cheese. J Brew Distilling 2012;3(2):15-22.

31. Pellegrini O, Remeuf F, Rivemale M, Barillet F. Renneting properties of milk from individual ewes: influence of genetic and non-genetic variables, and relationship with physico-chemical characteristics. J Dairy Res 1997;(64):355-366.

32. Alipanah ML, Kalashnikova A. Influence of k-casein genetic variant on cheese making ability. J Anim Vet Adv 2007;6(7):855-857.

33. Requena FD, Aguera EI, Requena F. Genética de la caseína de la leche en el bovino Frison. Rev Elec Vet 2007;(1):1-9.

34. Joudu I, Henno M, Värv S, Viinalass H, Püssa T, Kaart T, et al. The effect of milk proteins on milk coagulation properties in Stonian dairy breeds. Vet Zootech 2009;46(68):14-19.

35. Micari P, Caridi A, Colacino T, Caparra P, Cufari A. Physicochemical, microbiological and coagulating properties of ewe's milk produced on the Calabrian Mount Poro plateau. Int J Dairy Technol 2002;(55):204-210.

36. Barłowska J, Litwińczuk Z, Brodziak A, Chabuz W. Effect of the production season on nutritional value and technological suitability of milk obtained from intensive (tmr) and traditional feeding system of cows. J Microbiol Biotechnol Food Sci 2012;1(5):1205-1220.

37. Tyriseva AM, Vahlsten T, Ruottinen O, Ojala M. Noncoagulation of milk in Finnish Ayrshire and Holstein-Friesian cows and effect of herds on milk coagulation ability. J Dairy Sci 2004;(87):3958-3966.

38. Toffanin V, De Marchi M, Penasa M, Pretto D, Cassandro M. Characterization of milk coagulation ability in bulk milk samples. Acta Argicul Slove Suppl 2012;(3):93-98. 
39. Cecchinato A, Penasa M, Cipolat GC, De Marchi M, Bittante G. Short communication: Factors affecting coagulation properties of Mediterranean buffalo milk. J Dairy Sci 2011;(95):1709-1713.

40. Catarino I, Martins APL, Duarte E, Elane SP, Norberta de Pinho M. Rennet coagulation of sheep milk processed by ultrafiltration at low concentration factors. J Food Eng 2013;(114):249-254.

41. Auldist MJ, Johnston KA, White NJ, Fitzsimons WP, Boland MJ. A comparison of the composition, coagulation characteristics and cheesemaking capacity of milk from Friesian and Jersey dairy cows. J Dairy Res 2004;(71):51-57.

42. Pianaccioli L, Acciaioli A, Malvezzi R, Giustini L. Effect of season on characteristics of Pecorino cheese and Ricotta of Pistoiese Appennine: First results. Ital J Anim Sci 2007;(6):585-587.

43. Jaeggi JJ, Wendorff WL, Romero J, Berger M, Johnson ME. Impact of seasonal changes in ovine milk on composition and yield of a hard-pressed cheese. J Dairy Sci 2005;(88):1358-1363. 